\title{
Symmetric operations for all primes and Steenrod operations in Algebraic Cobordism
}

\author{
Alexander Vishik*
}

\begin{abstract}
In this article we construct Symmetric operations for all primes (previously known only for $p=2$ ). These unstable operations are more subtle than the Landweber-Novikov operations, and encode all $p$-primary divisibilities of characteristic numbers. Thus, taken together (for all primes) they plug the gap left by the Hurewitz map $\mathbb{L} \hookrightarrow \mathbb{Z}\left[b_{1}, b_{2}, \ldots\right]$, providing an important structure on Algebraic Cobordism. Applications include: questions of rationality of Chow group elements - see [13], and the structure of the Algebraic Cobordism - see [16. We also construct Steenrod operations of T.tom Dieck-style in Algebraic Cobordism. These unstable multiplicative operations are more canonical and subtle than Quillen-style operations, and complement the latter.
\end{abstract}

\section{Contents}

1 Introduction $\quad 2$

2 Algebraic Cobordism of Levine-Morel $\quad 3$

2.1 Generalized oriented cohomology theories . . . . . . . . . . . . . . 3

2.2 Formal group law . . . . . . . . . . . . . . . . . . . 4

3 Operations 4

3.1 General facts . . . . . . . . . . . . . . . . . . 4

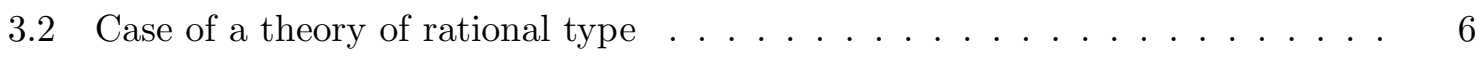

4 Two types of Steenrod operations on Cobordisms

5 Continuous group action on a power series ring 9

6 T.tom Dieck-style Steenrod operations 11

7 Symmetric operations for all primes 13

7.1 Construction . . . . . . . . . . . . . . . . . 13

7.2 Some properties. . . . . . . . . . . . . . . . 14

${ }^{*}$ School of Mathematical Sciences, University of Nottingham 


\section{Introduction}

The standard tool to distinguish and classify algebraic varieties is the use of various cohomology theories. The prominent place among them is occupied by Chow groups $\mathrm{CH}^{*}$ and $K_{0}$. Many results and conjectures in Algebraic Geometry are formulated in terms of these theories. But as was shown by M.Levine and F.Morel, these two theories are just small "faces" of much larger Algebraic Cobordism theory $\Omega^{*}$, which is an algebro-geometric analogue of the complex-oriented cobordism $M U^{*}$ in topology. This theory is rich because of the abundance of cohomological operations on it. The stable ones among them are provided by the Landweber-Novikov operations (see [4, Example 4.1.25]). These operations permit to prove (as well as to formulate!) many interesting results on Algebraic Cobordism, on Chow groups, and on $K_{0}$. But for some sorts of questions (related to torsion effects) these operations are not subtle enough. The remedy is the use of unstable operations. As the first example of such operations on $\Omega^{*}$, the Symmetric operations for $p=2$ were constructed in [11] and [12]. In [13] these operations were applied to the question of rationality of the Chow group elements, and they provide the only known way to deal with the 2-torsion there. More generally, it was observed that these operations control all 2-primary divisibilities of characteristic numbers, and thus plug 2-adically the gap between $\mathbb{L}=\Omega^{*}(\operatorname{Spec}(k))=\pi_{*}(M U)$ and $\mathbb{Z}\left[b_{1}, b_{2}, \ldots\right]=H_{*}(M U)$ left by the Hurewitz map. In Topology an analogous observation is (implicitly) contained in the beatiful work [8] of D.Quillen, where some traces of the topological counterparts of the mentioned operations are used. As soon as one realizes that Symmetric operations (for $p=2$ ) of [12] (constructed with a completely different purpose in mind) are analogous to operations used by D.Quillen to describe $M U^{*}(p t)$, the natural desire appears to control the mentioned "gap" p-adically for odd primes as well, taking into account that in Topology D.Quillen deals with such primes in the same way as with $p=2$. The needed Total Symmetric operation for the given $p$ must be the "negative part" of the (Quillen's type) Total Steenrod operation mod $p$ divided by "formal $p$ ". The problem though is to divide canonically. The obstacle here is that in our context we are deprived of the standard topological tools, and have to work not with spectra, but with cohomology theories themselves. The case $p=2$ was done by an explicit geometric construction (using $\mathrm{Hilb}_{2}$ ), and it is not clear at all how to generalize it. Besides, there existed no general methods to construct unstable operations in algebraic geometry, and aside from classical Adams operations in $K_{0}$ and mentioned Symmetric operations for $p=2$ no examples were known. After several years of attempts to construct Symmetric operations for arbitrary $p$, the author finally found an approach which permitted to describe all unstable additive operations in $\Omega^{*}$ and all theories obtained from it by change of coefficients - see [14]. In this approach, to construct an operation, one only needs to define it on the powers of the $\mathbb{P}^{\infty}$, and check that some compatibility rules are satisfied - see Theorem 3.6. One of the consequences is that we can "divide" operations canonically, since $\left(\mathbb{P}^{\infty}\right)^{\times r}$ is cellular, and $\mathbb{L}$ is an integral domain. This way, we obtain Symmetric operations for all primes - see Theorem 7.1 .

We also construct Steenrod operations of T.tom Dieck-style in Algebraic Cobordism. These operations are "more canonical" than Quillen-style Steenrod operations (in contrast to the latter, they depend on $p$ only). And while the Quillen-style Total Steenrod operation is just a specialization of the Total Landweber-Novikov operation, the one of the T.tom Dieck- 
style is an unstable multiplicative operation, so a much more subtle object. Our construction uses some derivatives of the Theorem 3.6 and a nice Theorem 5.6 describing the invariants of the continuous group action on a power series ring.

This text is organized as follows: In Section 2 we provide basic facts about Algebraic Cobordism and other Generalized oriented cohomology theories. In Section 3 we discuss cohomological operations between theories and introduce the notion of a theory of rational type. This class of theories contains $\Omega^{*}, \mathrm{CH}^{*}$ and $K_{0}$ and permits a complete description of the set of additive cohomological operations (obtained in [14]). In Section 4 we compare Steenrod operations of Quillen and T.tom Dieck-styles in Cobordisms. In Section 5 the continuous group action on the power series ring is studied. In Section 6 the results of Sections 3 and 6 are applied to produce the Steenrod operations of T.tom Dieck-style. And, finally, in Section 7 we construct Symmetric operations for all primes $p$, and deduce some properties of them.

Acknowledgements: I want to thank P.Brosnan, S.Gille, O.Haution, A.Lazarev, M.Levine, F.Morel, I.Panin, M.Rost, A.Smirnov, V.Voevodsky and other people for very useful conversations. The support of EPSRC Responsive Mode grant EP/G032556/1 is gratefully acknowledged. I'm grateful to the Referee for various suggestions and remarks which improved the exposition.

\section{Algebraic Cobordism of Levine-Morel}

\subsection{Generalized oriented cohomology theories}

In this article, $k$ will denote a field of characteristic zero, and $\mathbf{S m}_{\mathbf{k}}$ the category of smooth quasi-projective variaties over $k$. The notion of generalized oriented cohomology theory in Algebraic Geometry is borrowed from Topology (D.Quillen - [8]) with some variations.

Such a theory assigns to each smooth quasi-projective variety $X$ a (commutative and, possibly, graded) ring $A^{*}(X)$, together with the structure of pull-backs $f^{*}: A^{*}(Y) \rightarrow A^{*}(X)$ for all maps $f: X \rightarrow Y$, and the structure of push-forwards $f_{*}: A_{*}(X) \rightarrow A_{*}(Y)$ for all projective maps of constant relative dimension (where $A_{*}(X):=A^{\operatorname{dim}(X)-*}(X)$ for equidimensional $X$ ). These must satisfy certain set of axioms. We will use the definition of [14, Definition 2.1] which is the definition of M.Levine-F.Morel ([4, Definition 1.1.2]) plus the localization (excision) axiom (EXCI). So, everywhere below under "cohomology theory" we will mean a theory satisfying [14, Definition 2.1].

In [4] M.Levine and F.Morel constructed a universal generalized oriented cohomology theory $\Omega^{*}$ on $\mathbf{S m}_{\mathbf{k}}$ called Algebraic Cobordism (see [5] for an alternative definition). For a smooth quasi-projective $X$, the ring $\Omega^{*}(X)$ is additively generated by the classes $[V \stackrel{v}{\rightarrow} X]$ of projective maps with smooth $V$, modulo certain relations. This theory has a unique morphism of theories $\Omega^{*} \rightarrow A^{*}$ to any other theory $A^{*}$. If $k$ has a complex embedding, there is a natural topological realization functor $\Omega^{*}(X) \rightarrow M U^{2 *}(X(\mathbb{C}))$ which is an isomorphism for $X=\operatorname{Spec}(k)$. In the case of Chow groups, the natural morphism $\Omega^{*} \rightarrow \mathrm{CH}^{*}$ is surjective, and moreover, $\mathrm{CH}^{*}(X)=\Omega^{*}(X) \otimes_{\mathbb{L}} \mathbb{Z}$ ([4, Theorem 1.2.18]). The same is true about $K_{0}$ by

[4, Theorem 1.2.19]. Thus, Chow groups and $K_{0}$ can be reconstructed out of $\Omega^{*}$.

Since we will not work with the axioms, we will not reproduce them here, but we mention 
that any theory which is obtained from Algebraic Cobordism of Levine-Morel by change of coefficients: $A^{*}=\Omega^{*} \otimes_{\mathbb{L}} A$ is a theory in our sense. These are the free theories in the sense of M.Levine-F.Morel ([4, Remark 2.4.14]), and are exactly the theories of rational type of [14] (see [14, Proposition 4.9]). In particular, the theories $\Omega^{*}, \mathrm{CH}^{*}$ and $K_{0}$ are such.

\subsection{Formal group law}

Any theory in the above sense has Chern classes: a set of elements $c_{i}^{A}(\mathcal{V}) \in A^{i}(X)$ assigned to each vector bundle $\mathcal{V}$ on $X$, which satisfy the Cartan formula, and in the case of a linear bundle $\mathcal{L}, c_{1}^{A}(\mathcal{L})=s^{*} s_{*}(1)$, where $s: X \rightarrow \mathcal{L}$ is a zero section (see [7] or [6])). By [4, Theorem 2.3.13], any theory $A^{*}$ as above satisfies the axiom:

$(D I M)$ For any line bundles $\mathcal{L}_{1}, \ldots, \mathcal{L}_{n}$ on a smooth $X$ of dimension $<n$, one has: $c_{1}^{A}\left(\mathcal{L}_{1}\right) \cdot \ldots \cdot c_{1}^{A}\left(\mathcal{L}_{n}\right)=0 \in A_{*}(X)$.

Thus, any power series in Chern classes can be evaluated on any element of $A_{*}(X)$.

To any generalized oriented cohomology theory $A^{*}$ one can associate the Formal Group Law $\left(A, F_{A}\right)$. Here $A$ is the coefficient ring of $A^{*}$, and

$$
F_{A}(x, y)=\operatorname{Segre}^{*}(t) \in A[[x, y]]=A^{*}\left(\mathbb{P}^{\infty} \times \mathbb{P}^{\infty}\right),
$$

where $\mathbb{P}^{\infty} \times \mathbb{P}^{\infty} \stackrel{\text { Segre }}{\longrightarrow} \mathbb{P}^{\infty}$ is the Segre embedding, and $x, y, t$ are the 1-st Chern classes of $\mathcal{O}(1)$ of the respective copies of $\mathbb{P}^{\infty}$ (recall, that due to the projective bundle axiom, $\left.A^{*}\left(\mathbb{P}^{\infty}\right)=\lim _{\longleftarrow} A^{*}\left(\mathbb{P}^{n}\right)=A[[t]]\right)$. Denoting the coefficients of $F_{A}$ as $a_{i, j}^{A}$, we get:

$$
F_{A}(x, y)=\sum_{i, j} a_{i, j}^{A} \cdot x^{i} \cdot y^{j}
$$

The formal group law describes how to compute the 1-st Chern class of a tenzor product of two line bundles in terms of the 1-st Chern classes of factors:

$$
c_{1}^{A}(\mathcal{L} \otimes \mathcal{M})=F_{A}\left(c_{1}^{A}(\mathcal{L}), c_{1}^{A}(\mathcal{M})\right)
$$

The universal formal group law $\left(\mathbb{L}, F_{U}\right)$ has canonical morphism to any other formal group law, in particular, to $\left(A, F_{A}\right)$. M.Levine and F.Morel have shown that, in the case of algebraic cobordism, the respective map is an isomorphism - see [4, Theorem 1.2.7]. In particular, for any field $k, \Omega^{*}(k)=\mathbb{L}$ - the Lazard ring. As an abstract graded ring, $\mathbb{L} \cong \mathbb{Z}\left[x_{1}, x_{2}, \ldots\right]$, where $\operatorname{deg}\left(x_{i}\right)=i$.

\section{Operations}

\subsection{General facts}

To study cohomology theories effectively one needs a reasonable notion of "morphisms" between them. If we restrict ourselves to maps respecting both pull-backs and push-forwards, then there will be not many of such (for example, there will be only one map $\Omega^{*} \rightarrow A^{*}$, for any $A^{*}$ ). So, we have to permit more flexibility. The experience of Topology suggests that the right thing is to require that only pull-backs are respected. 
Definition 3.1 Let $A^{*}$ and $B^{*}$ be theories in the above sense. An operation $G: A^{n} \rightarrow B^{m}$ is a morphism of contravariant functors of sets pointed by 0 (in other words, a transformation commuting with the pull-backs, and sending zero to zero). An operation is called additive, if it is a homomorphism of abelian group. An operation $G: A^{*} \rightarrow B^{*}$ is called multiplicative, if it is a homomorphism of rings.

To each multiplicative operation one can assign certain power series - the inverse Todd genus $\gamma_{G}=b_{0} \cdot x+b_{1} \cdot x^{2}+b_{2} \cdot x^{3}+\ldots \in B[[x]]$, where, for $x^{A}=c_{1}^{A}(\mathcal{O}(1)), x^{B}=c_{1}^{B}(\mathcal{O}(1))$, one has: $G\left(x^{A}\right)=\gamma_{G}\left(x^{B}\right) \in B\left[\left[x^{B}\right]\right]=B\left(\mathbb{P}^{\infty}\right)$. Also, we have $\varphi_{G}: A \rightarrow B$ - the homomorphism of coefficient rings. The pair $\left(\varphi_{G}, \gamma_{G}\right)$ is a morphism of formal group laws: $\left(A, F_{A}\right) \longrightarrow\left(B, F_{B}\right)$. In other words,

$$
\varphi_{G}\left(F_{A}\right)\left(\gamma_{G}(u), \gamma_{G}(v)\right)=\gamma_{G}\left(F_{B}(u, v)\right)
$$

Of course, the composition of multiplicative operations corresponds to the composition of morphisms of formal group laws:

$$
\left(\varphi_{H \circ G}, \gamma_{H \circ G}(x)\right)=\left(\varphi_{H} \circ \varphi_{G}, \varphi_{H}\left(\gamma_{G}\right)\left(\gamma_{H}(x)\right)\right) .
$$

In the case of $A^{*}=\Omega^{*}$, and $b_{0}$ invertible in $B$, the homomorphism $\varphi_{G}$ is completely determined by $\gamma_{G}$. Namely, $\mathbb{L}$ is generated as a ring by universal coefficients $a_{i, j}^{\Omega}$, and $\varphi_{G}\left(a_{i, j}^{\Omega}\right)$ is the respective coefficient of the formal group law $F_{B}^{\gamma_{G}}(x, y)=\gamma_{G}\left(F_{B}\left(\gamma_{G}^{-1}(x), \gamma_{G}^{-1}(y)\right)\right)$. Moreover, from the reorientation procedure of I.Panin-A.Smirnov (see [7], 6], [10]) and universality of $\Omega^{*}$ of M.Levine-F.Morel (see [4, Theorem 1.2.6]) one obtains:

Theorem 3.2 (Panin-Smirnov+Levine-Morel) If $b_{0}$ is invertible in $B$, then for each $\gamma=$ $b_{0} x+b_{1} x^{2}+b_{2} x^{3}+\ldots \in B[[x]]$, there exists unique multiplicative operation $G: \Omega^{*} \rightarrow B^{*}$ with $\gamma_{G}=\gamma$.

One can easily introduce the notion of a stable operation - see (for example) [14, Definition 3.4]. As in Topology, these are operations commuting with some sort of suspension. To define the latter we need to consider theories on pairs $(X, U)$ where $U$ is an open subvariety of a smooth variety $X$. Fortunately, every theory in our sense naturally extends to pairs by the rule: $A^{*}((X, U)):=\operatorname{Ker}\left(A^{*}(X) \rightarrow A^{*}(U)\right)$, where we have to admit the non-unital rings into the game. And our suspension is just the smash-product with $\left(\mathbb{P}^{1}, \mathbb{P}^{1} \backslash\{0\}\right)$ - see [14]. An operation extends naturally to pairs as well, as long as it is "pointed" (sends 0 to 0 ). Hence, we can talk about stability. We should mention the following simple result (see, for example, [14, Proposition 3.9]):

Proposition 3.3 Let $G: A^{*} \rightarrow B^{*}$ be a multiplicative operation with $\gamma_{G}=b_{0} x+b_{1} x^{2}+\ldots$ Then $G$ is stable if and only if $b_{0}=1$.

The most important, and, in a sense, universal example of a stable multiplicative operation is provided by the Total Landweber-Novikov operation (see [4, Example 4.1.25]):

$$
S_{L-N}^{T o t}: \Omega^{*} \rightarrow \Omega^{*}\left[b_{1}, b_{2}, \ldots\right]
$$

with $\gamma_{S_{L-N}^{T o t}}(x)=x+b_{1} x^{2}+b_{2} x^{3}+\ldots$ Individual Landweber-Novikov operations are coefficients of the total one at particular $b$-monomials. 
Any stable multiplicative operation $G: \Omega^{*} \rightarrow B^{*}$ is a specialization of $S_{L-N}^{T o t}$. That is, $G=\eta \circ S_{L-N}^{T o t}$, where $\eta: \Omega^{*}\left[b_{1}, b_{2}, \ldots\right] \rightarrow B^{*}$ is a morphism of theories sending $b_{i}$ 's to the coefficients of $\gamma_{G}$. Similarly, any multiplicative operation as in Theorem 3.2 (i.e., with invertible $b_{0}$ ), is a generalized specialization of $S_{L-N}^{T o t}$. In other words, it is the composition of the reparametrization $\cdot b_{0}^{\text {codim }}: \Omega^{*} \rightarrow \Omega^{*}$ and the specialization as above corresponding to $\frac{\gamma_{G}}{b_{0}}-$ see [16, Section 3].

\subsection{Case of a theory of rational type}

Proposition 3.2 provides an effective tool in constructing stable operations from Algebraic Cobordism theory elsewhere. But in many situations one has to work with operations where $b_{0}$ is not invertible in the coefficient ring of the target theory $B^{*}$. The needed tools are provided by the results of [14] on theories of rational type.

These theories are defined in [14, Definition 4.1], but for us it will be important that these are exactly the free theories of M.Levine-F.Morel, that is, the theories obtained from $\Omega^{*}$ by change of coefficients: $A^{*}=\Omega^{*} \otimes_{\mathbb{L}} A$.

The principal result on multiplicative operations here is:

Theorem 3.4 ([14, Theorem 6.8]) Let $A^{*}$ be theory of rational type, and $B^{*}$ be any theory in the above sense. The assignment $G \leftrightarrow\left(\varphi_{G}, \gamma_{G}\right)$ defines a 1-to-1 correspondence between the set of multiplicative operations $A^{*} \stackrel{G}{\rightarrow} B^{*}$ and the set of homomorphisms $\left(A, F_{A}\right) \rightarrow\left(B, F_{B}\right)$ of the respective formal group laws.

This immediately gives an extension of Theorem 3.2 .

Theorem 3.5 ([14, Theorem 6.9]) Let $B^{*}$ be any theory in the above sense, and $b_{0} \in B$ be not a zero-divisor. Let $\gamma=b_{0} x+b_{1} x^{2}+b_{2} x^{3}+\ldots \in B[[x]]$. Then there exists a multiplicative operation $\Omega^{*} \stackrel{G}{\rightarrow} B^{*}$ with $\gamma_{G}=\gamma$ if and only if the shifted $F G L F_{B}^{\gamma} \in B\left[b_{0}^{-1}\right][[x, y]]$ has coefficients in $B$ (that is, has no denominators). In this case, such an operation is unique.

This result will enable us to construct the Steenrod operations of T.tom Dieck style below.

The methods of [14] and [15] permit to work with non-multiplicative operations as well. The main result (see also [14, Theorem 5.1] for the additive version) which implies all the rest is the following:

Theorem 3.6 ([15, Theorem 5.1]) Let $A^{*}$ be a theory of rational type, and $B^{*}$ be any theory in the above sense. Then the set of operation $A^{n} \stackrel{G}{\rightarrow} B^{*}$ on $\mathbf{S m}_{\mathbf{k}}$ is identified with the set of transformations

$$
A^{n}\left(\left(\mathbb{P}^{\infty}\right)^{\times l}\right) \stackrel{G}{\rightarrow} B^{*}\left(\left(\mathbb{P}^{\infty}\right)^{\times l}\right), \text { for } l \in \mathbb{Z}_{\geqslant 0}
$$

commuting with the pull-backs for:

(i) the action of the symmetric group $\mathfrak{S}_{l}$;

(ii) the partial diagonals; 
(iii) the partial Segre embeddings;

$(i v) \quad\left(\operatorname{Spec}(k) \hookrightarrow \mathbb{P}^{\infty}\right) \times\left(\mathbb{P}^{\infty}\right)^{\times r}, \forall r ;$

(v) the partial projections.

In Topology an analogous result was obtained by T.Kashiwabara - see [3, Theorem 4.2]. Under an additive subtheory $C^{*}$ of a theory $B^{*}$ we will mean an assignment $X \mapsto C^{*}(X)$, where $C^{*}(X) \subset B^{*}(X)$ is an additive subgroup, closed under pull-backs and push-forwards, and satisfying the axioms $(A 1),(A 2),(P B),(E H),(E X C I)$ of [14, Definition 2.1] (thus, only the axioms $(D 1),(D 2)$ are substituted by the notion of an additive subobject of such).

Theorem 3.6 immediately implies:

Proposition 3.7 Let $A^{*}$ be a theory of rational type, $B_{i}^{*}, i=1,2$ be any theories in our sense, and $C_{i}^{*} \subset B_{i}^{*}, i=1,2$ be additive subtheories. Let $Q: C_{1}^{*} \rightarrow C_{2}^{*}$ be an additive operation, such that $\left.Q\right|_{(\mathbb{P} \infty) \times l}$ is injective, for all $l \in \mathbb{Z}_{\geqslant 0}$, and $G: A^{n} \rightarrow C_{2}^{*}$ be an operation such that image $\left(\left.G\right|_{(\mathbb{P} \infty) \times l}\right) \subset \operatorname{image}\left(\left.Q\right|_{(\mathbb{P} \infty) \times l}\right)$, for all $l \in \mathbb{Z}_{\geqslant 0}$. Then there exists unique operation $H: A^{n} \rightarrow C_{1}^{*}$ such that $G=Q \circ H$.

Proof: By our condition, the transformation:

$$
A^{n}\left(\left(\mathbb{P}^{\infty}\right)^{\times l}\right) \stackrel{G}{\rightarrow} C_{2}^{*}\left(\left(\mathbb{P}^{\infty}\right)^{\times l}\right), \text { for } l \in \mathbb{Z}_{\geqslant 0}
$$

corresponding to $G$ can be written in a unique way as the composition of some transformation

$$
A^{n}\left(\left(\mathbb{P}^{\infty}\right)^{\times l}\right) \stackrel{H}{\rightarrow} C_{1}^{*}\left(\left(\mathbb{P}^{\infty}\right)^{\times l}\right), \text { for } l \in \mathbb{Z}_{\geqslant 0}
$$

and the operation $Q$. The fact that $H$-transformations will commute with all the pull-backs prescribed in the Theorem 3.6 follows from the respective property for $G$ and $Q$ together with the injectivity of $Q$. Hence, it can be extended to a unique operation $H: A^{n} \rightarrow C_{1}^{*}$ (a'priori we get an operation $H: A^{n} \rightarrow B_{1}^{*}$, but it lands in $C_{1}^{*}$, because it is so on $\left(\mathbb{P}^{\infty}\right)^{\times l}$, for all $l$ can be seen from the proof of Theorem 3.6). The fact that $G=Q \circ H$ is clear from the same Theorem 3.6

Corollary 3.8 Let $A^{*}$ be a theory of rational type, and $B^{*}$ be any theory in the above sense.

(1) Let $B^{*}=C^{*} \oplus D^{*}$ be an additive decomposition, and $b \in B$ be such an element, that the composition $m_{b}: C \stackrel{i_{C}}{\rightarrow} B \stackrel{\cdot b}{\rightarrow} B \stackrel{\pi_{C}}{\rightarrow} C$ is injective. Let $G: A^{n} \rightarrow B^{*}$ be an operation such that image $\left(\left.\pi_{C} \circ G\right|_{(\mathbb{P} \infty) \times l}\right) \subset \operatorname{image}\left(\left.m_{b}\right|_{(\mathbb{P} \infty) \times l}\right)$, for all $l \in \mathbb{Z}_{\geqslant 0}$. Then there exists unique operation $H: A^{n} \rightarrow C^{*} \hookrightarrow B^{*}$ such that

$$
(G-b \cdot H): A^{n} \rightarrow D^{*} \hookrightarrow B^{*}
$$

(2) Let $G: A^{n} \rightarrow B^{*}$ be an operation, and $b \in B$ be not a zero-divisor such that the image $\left(\left.G\right|_{(\mathbb{P} \infty) \times l}\right)$, for all $l \in \mathbb{Z}_{\geqslant 0}$ is divisible by $b$. Then there exists unique operation $H: A^{n} \rightarrow B^{*}$ such that $G=b \cdot H$. 
Proof: 1) Apply Proposition 3.7 with $B_{i}=B, C_{i}=C$, for $i=1,2, Q$ - the composition: $C^{*} \hookrightarrow B^{*} \stackrel{\cdot b}{\rightarrow} B^{*} \rightarrow C^{*}$ and $\pi_{C} \circ G: A^{n} \rightarrow C^{*}$. We obtain a unique operation $H: A^{n} \rightarrow C^{*}$ such that $\pi_{C} \circ G=Q \circ H$. Or, in other words, $(G-b \cdot H): A^{n} \rightarrow D^{*} \hookrightarrow B^{*}$.

2) Take $C^{*}=B^{*}$ and $D^{*}=0$ in (1).

\section{Two types of Steenrod operations on Cobordisms}

In Topology, all additive operations on singular homology modulo $p$ are generated by the Steenrod operations (and Bockstein). These can be organized into a multiplicative Total Steenrod operation, which can be constructed as follows. Denoting as $H_{\mathbb{Z} / p}^{*}$ - the $\mathbb{Z} / p$ equivariant singular homology, one gets a natural map

$$
H^{m}(X, \mathbb{Z} / p) \rightarrow H_{\mathbb{Z} / p}^{p m}\left(X^{\times p}, \mathbb{Z} / p\right) \rightarrow H_{\mathbb{Z} / p}^{p m}(X, \mathbb{Z} / p),
$$

where the last arrow is induced by the diagonal embedding $X \stackrel{\Delta}{\rightarrow} X^{\times p}$. And since the $\mathbb{Z} / p$ action on $X$ is trivial, the target group can be identified with $H^{p m}(X \times \mathrm{B} \mathbb{Z} / p, \mathbb{Z} / p)$, which is the component of degree $p m$ of $H^{*}(X, \mathbb{Z} / p) \otimes_{\mathbb{Z} / p} H^{*}(\mathrm{~B} \mathbb{Z} / p, \mathbb{Z} / p)=H^{*}(X, \mathbb{Z} / p)[[t]][y] /\left(y^{2}-c\right)$, where $\operatorname{deg}(y)=1, \operatorname{deg}(t)=2$, and $c=0$, for $p>2$, and $c=t$, for $p=2$. One obtains a multiplicative operation

$$
S q: H^{*}(X, \mathbb{Z} / p) \rightarrow H^{*}(X, \mathbb{Z} / p)[[t]][y] /\left(y^{2}-c\right),
$$

whose only non-trivial components at monomials in $t$ are of degrees divisible by $(p-1)$. These are the individual Steenrod operations. P.Brosnan has shown that the above construction goes through in the algebro-geometric context (for Chow groups modulo $p$ ) as well - see [1] (in a more general case of motivic cohomology these operations were produced previously by V.Voevodsky by a different construction - see [17]).

Steenrod operations on singular cohomology modulo prime can be extended to the theory of complex-oriented cobordisms $M U^{*}$ in (at least) two ways.

The first construction due to T.tom Dieck ([2]) uses the same geometric approach with the $\mathbb{Z} / p$-equivariant cohomology replaced by the $\mathbb{Z} / p$-equivariant cobordism, and gives the multiplicative operation:

$$
S q: M U^{*}(X) \rightarrow M U^{*}(X \times \mathrm{B} \mathbb{Z} / p)=M U^{*}(X)[[t]] /(p \cdot M U t) \rightarrow M U^{*}(X)[[t]] /\left(\frac{p \cdot M U t}{t}\right) .
$$

Note, in particular, that one gets a completely canonical operation depending on $p$ only.

The second construction due to D.Quillen ([8]) is based on the universal property of complex-oriented cobordism which implies that any power series $\gamma=b_{0} x+b_{1} x^{2}+b_{2} x^{3}+\ldots$ with $b_{0} \in B$ invertible corresponds to a unique multiplicative operation $G: M U^{*} \rightarrow B^{*}$. It remains to specify $B^{*}$ and $\gamma$. One chooses representatives $\left\{i_{j}, 0<j<p\right\}$ of all nonzero cosets modulo $p$, and defines $\gamma=x \prod_{j=1}^{p-1}\left(x+{ }_{M U} i_{j} \cdot M U t\right) \in \mathbb{L}[[t]][[x]]$. This gives a multiplicative operation:

$$
S t(\bar{i}): M U^{*} \rightarrow M U^{*}\left[\mathbf{i}^{-1}\right][[t]]\left[t^{-1}\right]
$$


where $\mathbf{i}:=\prod_{j=1}^{p-1} i_{j}$. Note, that this operation depends on the choice of coset representatives (of course, one can take $i_{j}=j$, for $j=1, \ldots, p-1$, but for general $p$, such a choice will be about as good as any other). As was shown by D.Quillen, his operation agrees with the one of T.tom Dieck. Namely, there is the following commutative diagram:

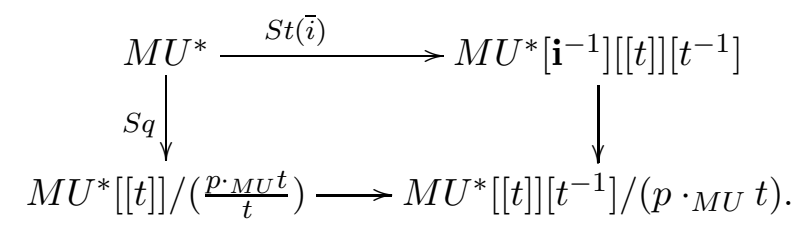

The version of D.Quillen can be easily extended to the Algebraic Cobordism of M.LevineF.Morel using the universality of $\Omega^{*}$ ([4, Theorem 1.2.6]). So, one gets a multiplicative operation

$$
S t(\bar{i}): \Omega^{*} \rightarrow \Omega^{*}\left[\mathbf{i}^{-1}\right][[t]]\left[t^{-1}\right],
$$

with the same $\gamma$ as above. The situation with the version of T.tom Dieck is more delicate. It is easy to define the $\mathbb{Z} / p$-equivariant Algebraic Cobordism, but the problems appear when one tries to show that the natural map $\Omega^{m}(X) \rightarrow \Omega_{\mathbb{Z} / p}^{p m}\left(X^{\times p}\right)$ is well-defined. The reason is that the defining relations in the Algebraic Cobordism theory are more complicated than in the complex-oriented cobordism. Namely, aside from the usual elementary cobordism relations one has also the double point relations - see [5]. It is rather easy to show that the elementary cobordism relations are respected by our map, but the author was unable to do the double point case. And although the author succeeded for $p=2$, he had to employ the Symmetric operations modulo 2. Until now these operations were unavailable for $p>2$, and one of the principal aims of the current article is to construct them. So, we have to use a different approach. Fortunately, the methods of [14] give us all the necessary tools.

\section{Continuous group action on a power series ring}

To deal with the T.tom Dieck-style Steenrod operations we will need to compute the invariants of the continuous action of a finite group on a power series ring.

The following Lemma is the key to such a description.

Lemma 5.1 Let $R=\lim _{\leftarrow} R_{n}$ be a commutative ring, and $t_{1}, \ldots, t_{r} \in R$ be such elements that $R$ is complete with respect to $t_{i}$ (that $i s, t_{i}^{n}=0 \in R_{n}$ ). Suppose $t_{i}$ and $\left(t_{i}-t_{j}\right)$, for $i \neq j$ are not zero-divisors in $R$. Let $k, n_{1}, \ldots, n_{r} \in \mathbb{N}$, and $f(x)=\sum_{l \geqslant k} \alpha_{l} x^{l} \in R[[x]]$ be such power series that $f\left(t_{i}+y\right)=\sum_{j \geqslant n_{i}} \beta_{i, j} y^{j}$, for some $\beta_{i, j} \in R$. Then $\alpha_{k}$ is divisible by $\prod_{i=1}^{r} t_{i}^{n_{i}}$.

Proof: Let $\left(n_{1}, \ldots, n_{r}\right)$ be an $r$-tuple of natural numbers, and $m \geqslant N:=\sum_{j=1}^{r} n_{j}$. Denote $N_{i}:=\sum_{j=1}^{i} n_{j}$. For $1 \leqslant u \leqslant N$, denote as $|u|$ such $1 \leqslant i \leqslant r$ that $N_{i-1}<u \leqslant N_{i}$, and as $\bar{u}$ the difference $u-N_{i-1}-1$ (so, $\left.0 \leqslant \bar{u}<n_{|u|}\right)$. Consider $N \times m$ matrix $A\left(n_{1}, \ldots, n_{r} ; m\right)=\left\{a_{u, v}\right\}$, where $a_{u, v}=\left(\begin{array}{c}v-1 \\ \bar{u}\end{array}\right) t_{|u|}^{v-1-\bar{u}}$.

Claim 5.2 (1) All $N \times N$ minors of the matrix $A\left(n_{1}, \ldots, n_{r} ; m\right)$ are divisible by $\prod_{i>j}\left(t_{i}-t_{j}\right)^{n_{i} \cdot n_{j}}$ 


$$
\text { If } m=N \text {, then } \operatorname{det}\left(A\left(n_{1}, \ldots, n_{r} ; m\right)\right)=\prod_{i>j}\left(t_{i}-t_{j}\right)^{n_{i} \cdot n_{j}} .
$$

Proof: Let us prove both statements by induction on $N$. For $N=0$ there is nothing to prove. Notice, that elementary transformations on rows and columns do not change the ideal generated by minors, while multiplication of some row by $\lambda$ multiplies this ideal by $\lambda$. Perform the following transformations (in the prescribed order):

1) For all $m>v \geqslant 1$ subtract $v$-th column times $t_{1}$ from $(v+1)$-st.

2 ) For all $2 \leqslant i \leqslant r$ subtract 1 -st row from $\left(N_{i-1}+1\right)$-st, and divide the result (the new $\left(N_{i-1}+1\right)$-st row) by $\left(t_{i}-t_{1}\right)$.

3) For all $2 \leqslant i \leqslant r$, for all $N_{i-1}+1 \leqslant u<N_{i}$, subtract $u$-th row from $(u+1)$-st, and divide the result (the new $(u+1)$-st row) by $\left(t_{i}-t_{1}\right)$.

The result will be the block matrix $1 \times A\left(n_{1}-1, n_{2}, \ldots, n_{r} ; m-1\right)$. Thus, the ideal generated by the minors of $A\left(n_{1}, \ldots, n_{r} ; m\right)$ is $\prod_{i=2}^{r}\left(t_{i}-t_{1}\right)^{n_{i}}$ times the ideal generated by the minors of $A\left(n_{1}-1, n_{2}, \ldots, n_{r} ; m-1\right)$. Induction step is proven.

Since for $f(x)=\sum_{l \geqslant k} \alpha_{l} x^{l}$, we have $f\left(t_{i}+y\right)=\sum_{j \geqslant 0} \beta_{i, j} y^{j}$ with $\beta_{i, j}=0$, for $j<n_{i}$, we get $n_{i}$ equations:

$$
\sum_{l \geqslant k}\left(\begin{array}{c}
l \\
w
\end{array}\right) t_{i}^{l-w} \alpha_{l}=0, \quad 0 \leqslant w<n_{i} .
$$

Performing elementary transformations with rows and dividing by $t_{i}$ (which is possible since $t_{i}$ is not a zero divisor), we get the equivalent system:

$$
\sum_{l \geqslant k}\left(\begin{array}{c}
l-k \\
w
\end{array}\right) t_{i}^{l-k-w} \alpha_{l}=0, \quad 0 \leqslant w<n_{i}
$$

Combining all such systems for $1 \leqslant i \leqslant r$, we get the system with the matrix $A\left(n_{1}, \ldots, n_{r} ; \infty\right)$. Let $B$ be such a matrix that

$$
B \cdot A\left(n_{1}, \ldots, n_{r} ; N\right)=\operatorname{det}\left(A\left(n_{1}, \ldots, n_{r} ; N\right)\right) \cdot I d=\prod_{i>j}\left(t_{i}-t_{j}\right)^{n_{i} \cdot n_{j}} \cdot I d .
$$

Since $\left(t_{i}-t_{j}\right)$ are not zero divisors, the system with the matrix $A\left(n_{1}, \ldots, n_{r} ; \infty\right)$ is equivalent to the system with the matrix $B \cdot A\left(n_{1}, \ldots, n_{r} ; \infty\right)$. This shows that $\prod_{i>j}\left(t_{i}-t_{j}\right)^{n_{i} \cdot n_{j} \cdot \alpha_{k}}$ can be expressed as a linear combination of $\alpha_{l}$, with $l \geqslant k+N$. And, by the Kramer's rule, the coefficient at $\alpha_{k+m}$ will be (minus) the minor $M_{2,3, \ldots, N, m+1}$ of the matrix $A\left(n_{1}, \ldots, n_{r} ; \infty\right)$. Dividing by $t_{i}$ and performing elementary transformations with rows we see that this minor is equal to $\prod_{i=1}^{r} t_{i}^{n_{i}}$ times the minor $M_{1,2, \ldots, N-1, m}$. It follows from the Claim 5.2 that $M_{1,2, \ldots, N-1, m}$ is divisible by $\prod_{i>j}\left(t_{i}-t_{j}\right)^{n_{i} \cdot n_{j}}$. Using again the fact that $\left(t_{i}-t_{j}\right)$ is not a zero divisor, we express $\alpha_{k}$ as a linear combination of $\alpha_{l}, l \geqslant k+N$, where all the coefficients are divisible by $\prod_{i=1}^{r} t_{i}^{n_{i}}$.

Remark 5.3 Above we are working with infinite linear relations, but it follows from our condition on $t_{i}$ 's that the respective sums do converge. 
Definition 5.4 Let $B=\lim _{\leftarrow} B_{n}$ be a commutative ring. We say that $\sigma: B[[x]] \rightarrow B[[x]]$ is a continuous $B$-homomorphism, if, for any $\varepsilon(x) \in B[[x]]$, one has $\sigma(\varepsilon(x))=\varepsilon\left(x^{\sigma}\right)$, where $x^{\sigma}=\sum_{j \geqslant 0} \lambda_{j}^{\sigma} x^{j}$, and $B$ is complete with respect to $\lambda_{0}^{\sigma}$ (that is, $\left(\lambda_{0}^{\sigma}\right)^{n}=0 \in B_{n}$ ). If $\lambda_{1}^{\sigma} \in B$ is invertible, then such map has an inverse (also continuous B-homomorphism), and we call it continuous B-automorphism. Continuous B-automorphisms form a group $\operatorname{Aut}_{B}^{c}(B[[x]])$ under composition.

Definition 5.5 Let $G$ be a group. We say that $G$ acts continuously on $B[[x]]$ if we are given a group homomorphism $\rho: G \rightarrow \operatorname{Aut}_{B}^{c}(B[[x]])$.

Theorem 5.6 Let $\rho: G \rightarrow \operatorname{Aut}_{B}^{c}(B[[x]])$ be a continuous action of a finite group $G$ on $B[[x]]$. Suppose that for all $g \in G \backslash e$, the elements $\lambda_{0}^{g} \in B$ are not zero divisors. Then the subring of invariants is given by:

$$
B[[x]]^{G}=B\left[\left[\prod_{g \in G} x^{g}\right]\right]
$$

Proof: Let $\varphi(x) \in B[[x]]^{G}$ be invariant power series. Let $\varphi(x)=\sum_{j \geqslant n} \alpha_{j} x^{j}$. Consider the power series $x^{g} \in B[[x]], g \in G$. Let us denote $t_{g}:=\lambda_{0}^{g}$.

Let $g, h \in G$ be different elements. Then $x^{g}=\left(x^{h}\right)^{h^{-1} g}$. Thus, $t_{g}=t_{h}+\sum_{j \geqslant 1} \lambda_{j}^{h} t_{h^{-1} g}^{j}$, and, up to an invertible factor, $\left(t_{g}-t_{h}\right)$ is equal to $t_{h^{-1} g}$, which is not a zero divisor.

Since $\varphi(x)=\varphi\left(x^{g}\right)=\varphi\left(t_{g}+y_{g}\right)$, where $y_{g}=\sum_{j \geqslant 1} \lambda_{j}^{g} x^{j}$, and the ideal $(x)$ generated by $x$ coincides with the ideal $\left(y_{g}\right)$ generated by $y_{g}$ (since $\lambda_{1}^{g}$ is invertible), we have that $\varphi\left(t_{g}+y_{g}\right)=\sum_{j \geqslant n} \beta_{g, j} y_{g}^{j}$. It follows from Lemma [5.1 that $\alpha_{n}$ is divisible by $\prod_{g \in G \backslash e} t_{g}^{n}$. Let $\gamma_{n} \cdot \prod_{g \in G \backslash e} t_{g}^{n}=\alpha_{n}$. Then $\psi(x):=\varphi(x)-\gamma_{n} \cdot\left(\prod_{g \in G} x^{g}\right)^{n}$ is also invariant, and belongs to $(x)^{n+1}$.

Thus, any power series invariant under $G$ can be expressed as a power series in $\left(\prod_{g \in G} x^{g}\right)$. Theorem is proven.

Corollary 5.7 Let $B$ be commutative ring, with a continuous action of $\mathbb{Z} / p \cdot\langle\sigma\rangle$ (p-prime) on $B[[x]]$. Suppose that $t_{\sigma} \in B$ is not a zero divisor. Then

$$
B[[x]]^{\mathbb{Z} / p}=B\left[\left[\prod_{i=0}^{p-1} x^{\sigma^{i}}\right]\right] .
$$

Proof: It is sufficient to observe that $t_{g^{m}}$ is divisible by $t_{g}$. Since, for arbitrary non-zero element $g \in \mathbb{Z} / p \cdot\langle\sigma\rangle$, there is $m$ such that $g^{m}=\sigma$, we have that $t_{g}$ is not a zero divisor, and we can apply Theorem 5.6 .

\section{T.tom Dieck-style Steenrod operations}

Let $R$ be commutative ring with the formal group law on it (or, which is the same, with the ring homomorphism $\varepsilon: \mathbb{L} \rightarrow R$ ), and $p$ be prime number. Define $B:=R[[t]] /\left(\frac{p_{F} t}{t}\right)$, where 
$p \cdot{ }_{F} t \in R[[t]]$ is $p$ times $t$ in the sense of the formal group law. Then $B$ is complete with respect to $t$.

Let us define the continuous action of $\mathbb{Z} / p \cdot\langle\sigma\rangle$ on $B[[x]]$ by the formula: $x^{\sigma}=\left(x+{ }_{F} t\right) \in$ $B[[x]]$. Notice, that $t_{\sigma}=t$, and $\lambda_{1}^{\sigma}=1+\sum_{i \geqslant 1} a_{i, 1} t^{i}$ is invertible. Suppose $p$ is not a zero divisor in $R$. Then $t$ is not a zero divisor in $B$, and we can apply Corollary 5.7, We get:

Corollary 6.1 In the above situation,

$$
B[[x]]^{\mathbb{Z} / p}=B\left[\left[\prod_{i=0}^{p-1}\left(x+{ }_{F} i \cdot_{F} t\right)\right]\right] .
$$

Proposition 6.2 Let $R$ be commutative ring with the formal group law, and $B:=R[[t]] /\left(\frac{p \cdot F t}{t}\right)$, then there exists power series in two variables $G(u, v)$ with coefficients in $B$ such that

$$
\prod_{i=0}^{p-1}\left(x+{ }_{F} y+{ }_{F} i \cdot_{F} t\right)=G\left(\prod_{i=0}^{p-1}\left(x+{ }_{F} i \cdot_{F} t\right), \prod_{i=0}^{p-1}\left(y+{ }_{F} i \cdot_{F} t\right)\right) .
$$

Proof: Clearly, it is sufficient to prove this statement for $R=\mathbb{L}$ with the universal formal group law on it. In this case, $p$ is not a zero divisor in $R$, and so $t$ is not a zero-divisor in $B$. Consider the action of $\mathbb{Z} / p \times \mathbb{Z} / p$ on $B[[x, y]]$ given by $x^{\sigma}=\left(x+{ }_{F} t\right), y^{\sigma}=y, x^{\tau}=x$, $y^{\tau}=\left(y+{ }_{F} t\right)$. Clearly, $\prod_{i=0}^{p-1}\left(x+_{F} y+_{F} i \cdot_{F} t\right) \in B[[x, y]]^{\mathbb{Z} / p \times \mathbb{Z} / p}$. Applying Corollary 6.1, we obtain: $B[[x, y]]^{\mathbb{Z} / p \times 1}=B\left[\left[\prod_{i=0}^{p-1}\left(x+{ }_{F} i \cdot_{F} t\right), y\right]\right]=: C[[y]]$, and $t$ is not a zero divisor in $C$ either. Thus,

$$
B[[x, y]]^{\mathbb{Z} / p \times \mathbb{Z} / p}=B\left[\left[\prod_{i=0}^{p-1}\left(x+{ }_{F} i \cdot \cdot_{F} t\right), \prod_{i=0}^{p-1}\left(y+{ }_{F} i \cdot{ }_{F} t\right)\right]\right] .
$$

Proposition is proven.

In the above situation (with $t \in B$ not a zero-divisor), consider the power series $\alpha(x)=$ $x \prod_{i=1}^{p-1}\left(x+{ }_{F} i \cdot{ }_{F} t\right) \in B[[x]]$. The first term of this power series is $\left(\prod_{i=1}^{p-1} i \cdot{ }_{F} t\right) \cdot x$. Notice, that $i \cdot{ }_{F} t$, for $i=1, \ldots, p-1$, are invertible in $B\left[t^{-1}\right]$. Thus, there exists the inverse power series $\beta(y) \in B\left[t^{-1}\right][[y]]$ such that $\beta(\alpha(x))=x$.

Consider the twisted formal group law $F^{\alpha}$ given by

$$
F^{\alpha}(u, v):=\alpha(F(\beta(u), \beta(v))) \in B\left[t^{-1}\right][[u, v]]
$$

Proposition 6.3 The formal group law $F^{\alpha}$ has coefficients in B.

Proof: This follows immediately from Proposition 6.2.

Combining this with Theorem 3.5 we obtain Steenrod operations of T.tom Dieck style for $\Omega^{*}$ : 
Theorem 6.4 For each prime $p$ there exists (unique) multiplicative operation

$$
S q: \Omega^{*} \rightarrow \Omega^{*}[[t]] /\left(\frac{p \cdot \Omega t}{t}\right)
$$

with $\gamma_{S q}(x)=x \cdot \prod_{0<i<p}(x+\Omega i \cdot \Omega t)$.

\section{Symmetric operations for all primes}

\subsection{Construction}

By comparing the respective morphisms of formal group laws and using Theorem 3.4 we obtain a commutative diagram relating Steenrod operations of D.Quillen and T.tom Dieck types:

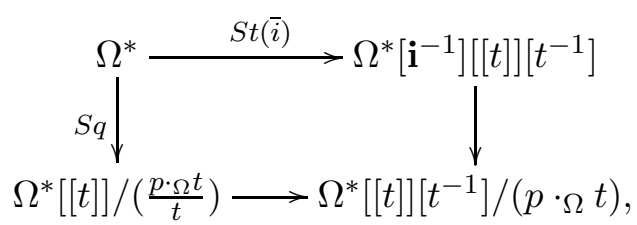

where $\bar{i}$ is any choice of coset representatives.

Since the target of $S q$ has no negative powers of $t$, and the $t^{0}$-component of it coincides with the $p$-th power $\square^{p}$, the commutativity of the above diagram shows that the non-positive part of $\left(\square^{p}-S t(\bar{i})\right)$ is divisible by $[p]_{\Omega}:=\frac{p \cdot \Omega t}{t}$. I should point out that this fact itself can be proven without Steenrod operations of T.tom Dieck type, and without the Theorem 3.4 (or methods of [14]). But what is much deeper, it appears that one can divide "canonically", and the quotient is what we call Symmetric operation.

Theorem 7.1 There is unique operation $\Phi(\bar{i}): \Omega^{*} \rightarrow \Omega^{*}\left[\mathbf{i}^{-1}\right]\left[t^{-1}\right]$ such that

$$
\left(\square^{p}-S t(\bar{i})-[p]_{\Omega} \cdot \Phi(\bar{i})\right): \Omega^{*} \rightarrow \Omega^{*}\left[\mathbf{i}^{-1}\right][[t]] t .
$$

Proof: Consider $A^{*}=\Omega^{*}, B^{*}=\Omega^{*}\left[\mathbf{i}^{-1}\right][[t]]\left[t^{-1}\right], C^{*}=\Omega^{*}\left[\mathbf{i}^{-1}\right]\left[t^{-1}\right]$, and $D^{*}=\Omega^{*}\left[\mathbf{i}^{-1}\right][[t]] t$. Take $b=[p]_{\Omega}$. Then $m_{b}: \mathbb{L}\left[\mathbf{i}^{-1}\right]\left[t^{-1}\right] \rightarrow \mathbb{L}\left[\mathbf{i}^{-1}\right]\left[t^{-1}\right]$ is injective as $\mathbb{L}$ is an integral domain. Consider $G=\left(\square^{p}-S t(\bar{i})\right): A^{*} \rightarrow B^{*}$. Then $\pi_{C} \circ G=S t(\bar{i})_{\leqslant 0}: A^{*} \rightarrow B^{*}$ - the nonpositive part of $\left(\square^{p}-S t(\bar{i})\right)$ corresponding to monomials in $t$ of non-positive degree. By the above diagram, image $\left(\pi_{C} \circ G\right) \subset$ image $\left(m_{b}\right)$, and by Corollary 3.8, there is unique operation $\Phi(\bar{i}): A^{*} \rightarrow C^{*}$ such that $\left(\square^{p}-S t(\bar{i})-b \cdot \Phi(\bar{i})\right): A^{*} \rightarrow D^{*}$.

Some traces of the $M U$-analogue of this operation were used by D.Quillen in [8], and they provide the main tool of the mentioned article.

In Algebraic Cobordism the described operation appeared originally in the works [11] and [12] of the author in the case $p=2$ in a different form. Namely, in the form of "slices", which were constructed geometrically. Only substantially later the author had realized that these slices can be combined into the "formal half" of the "negative part" of some multiplicative 
operation, which had a power series $\gamma=x \cdot(x-\Omega t)$ reminiscent of a Steenrod operation in Chow groups mod 2.

Out of our operation $\Phi(\bar{i})$ we would like to produce some maps from $\Omega^{*}$ to itself. The natural approach would be to consider the coefficients of it at particular monomials $t^{-n}$, or, which is close, $\operatorname{Res}_{t=0} \frac{t^{n} \cdot \Phi(\bar{i}) \omega_{t}}{t}$. Here $\omega_{t}$ is the canonical invariant (w.r.to our FGL) form $\left(\left[\mathbb{P}^{0}\right]+\left[\mathbb{P}^{1}\right] t+\left[\mathbb{P}^{2}\right] t^{2}+\ldots\right) d t$ - see $\left[14\right.$, Sect. 7.1], and $\operatorname{Res}_{t=0}$ is the coefficient at $\frac{d t}{t}$. And, if one thinks about it, there is no point restricting oneself to monomials, so one can consider

$$
\Phi(\bar{i})^{q(t)}:=\operatorname{Res}_{t=0} \frac{q(t) \cdot \Phi(\bar{i}) \omega_{t}}{t},
$$

where $q(t)=q_{0}+q_{1} t+q_{2} t^{2}+\ldots \in \mathbb{L}[[t]]$ is any power series. Of course, there are various relations among these slices which bind them together into the operation $\Phi(\bar{i})$. For $p=2$, these are exactly the Symmetric operations $\Phi^{q(t)}$ of [12]:

Proposition 7.2 In the case $p=2$, with $\bar{i}=\{-1\}$, for any power series as above, we have:

$$
\Phi(\bar{i})^{q(t)}=\Phi^{q(t)} .
$$

Proof: By Theorem 3.6, it is sufficient to compare our operations on cellular spaces $\left(\mathbb{P}^{\infty}\right)^{\times r}$. It follows from [12, Propositions 2.13 and 2.15] that, for any $q(t) \in \mathbb{L}[[t]], \Phi^{\left([2]_{\Omega}\right) q(t)}=$ $q(0) \cdot \square-\operatorname{Res}_{t=0} \frac{q(t) \cdot \Psi \cdot \omega_{t}}{t}$, where $\Psi: \Omega^{*} \rightarrow \Omega^{*}[[t]]\left[t^{-1}\right]$ is the multiplicative operation with $\gamma_{\Psi}=x \cdot(x-\Omega t)$. Thus, $\Psi=S t(\bar{i})$, where $\bar{i}=\{-1\}$. And so, $\Phi^{\left([2]_{\Omega}\right) q(t)}=\Phi(\bar{i})^{\left([2]_{\Omega}\right) q(t)}$, by the very definition of the latter. But on cellular spaces, the multiplication by $[2]_{\Omega}$ is injective, as $\Omega^{*}$ of such a space is a free $\mathbb{L}$-module (for $\left(\mathbb{P}^{\infty}\right)^{\times r}$, it is a direct consequence of the projective bundle axiom - see Subsect. 2.2). Hence, $\Phi(\bar{i})^{q(t)}=\Phi^{q(t)}$ as well (cf. [12, proof of Corollary $2.17])$.

The cases $p=2$ and 3 are special, since we can choose our representatives $\bar{i}$ to be invertible in $\mathbb{Z}$. For $p=2$, we have two such choices: $\{1\}$, or $\{-1\}$ (in [12], $\{-1\}$ was "chosen"). For $p=3$, the choice is canonical: $\{1,-1\}$. Thus, we get integral operations $\Phi(\bar{i}): \Omega^{*} \rightarrow \Omega^{*}\left[t^{-1}\right]$. And, for arbitrary $p$, representatives can be chosen as the powers of some fixed prime $l$ (generating $\left.(\mathbb{Z} / p)^{*}\right)$, so that only one prime would be inverted. Moreover, this prime can be selected in infinitely many ways, so, in a sense, the picture is as good as integral.

\subsection{Some properties}

First of all, we should mention the Riemann-Roch type result which describes how our operations behave with respect to regular embeddings.

Let $\mathcal{N}$ be a vector bundle on $X$ with $\Omega$-roots $\lambda_{1}, \ldots, \lambda_{d}$. Denote as $c^{\Omega}(\mathcal{N} ; \bar{i})$ the element

$$
\prod_{l=1}^{d}\left(\frac{\gamma_{S t(\bar{i})}(x)}{x}\right)\left(\lambda_{l}\right)=\prod_{l=1}^{d} \prod_{j=1}^{p-1}\left(\lambda_{l}+\Omega i_{j} \cdot \Omega t\right)=\prod_{j=1}^{p-1} c^{\Omega}(\mathcal{N})\left(i_{j} \cdot \Omega t\right) \in \Omega^{*}(X)[[t]],
$$

where $c^{\Omega}(\mathcal{N})(t)=\prod_{i=1}^{d}\left(t+\Omega \lambda_{l}\right)$. Analogously, one can define the Chow group versions: $c(\mathcal{N})(t)$ - the usual total Chern power series, and $c(\mathcal{N} ; \bar{i})$ (where the formal addition is substituted by the usual one). Then we have (cf. [12, Proposition 3.1]): 
Proposition 7.3 Let $X \stackrel{f}{\rightarrow} Y$ be a regular embedding of smooth quasi-projective varieties with the normal bundle $\mathcal{N}_{f}$, and $q(t)=q_{0}+q_{1} t+q_{2} t^{2}+\ldots \in \Omega^{*}(X)[[t]]$. Then

$$
\Phi(\bar{i})^{q(t)}\left(f_{*}(u)\right)=f_{*}\left(\Phi(\bar{i})^{q(t) \cdot c^{\Omega}\left(\mathcal{N}_{f} ; \bar{i}\right)}(u)\right) .
$$

Proof: Consider two operations:

$$
\Omega^{*}(Z) \stackrel{G, \widetilde{G}}{\longrightarrow} \Omega^{*}\left(Z \times\left(\mathbb{P}^{\infty}\right)^{\times d}\right)
$$

where $G(v):=\Phi(\bar{i})^{q(t)}\left(\pi^{*}(v) \cdot \prod_{l=1}^{d} z_{l}\right)$, and $\widetilde{G}(v):=\Phi(\bar{i})^{\widetilde{q}(t)}\left(\pi^{*}(v)\right)$, where $z_{l}=c_{1}^{\Omega}\left(\mathcal{O}(1)_{l}\right)$, $\pi: X \times\left(\mathbb{P}^{\infty}\right)^{\times r} \rightarrow X$ is the projection, and $\widetilde{q}(t)=q(t) \cdot \prod_{l=1}^{d} \gamma_{S t(\bar{i})}\left(z_{l}\right)$. We can write $\widetilde{q}(t)$ as $\widetilde{\widetilde{q}}(t) \cdot \prod_{l=1}^{d} z_{l}$, where $\widetilde{\widetilde{q}}(t)=q(t) \cdot \prod_{l=1}^{d}\left(\frac{\gamma_{S t(\bar{i})}(x)}{x}\right)\left(z_{l}\right)$. Operations $G$ and $\widetilde{G}$ coincide on all $\left(\mathbb{P}^{\infty}\right)^{\times m}$. This follows from: the fact that for the multiplicative operation $H=S t(\bar{i})$ we have: $H\left(\pi^{*}(v) \cdot \prod_{l=1}^{d} z_{l}\right)=H\left(\pi^{*}(v)\right) \cdot \prod_{l=1}^{d} \gamma_{S t(\bar{i})}\left(z_{l}\right)$; and the fact that on cellular spaces such as $\left(\mathbb{P}^{\infty}\right)^{\times m}, \Phi(\bar{i})^{r(t)}$ can be written as $\operatorname{Res}_{t=0} \frac{\left(r(0) \square^{p}-r(t) \cdot H\right) \omega_{t}}{p \cdot \Omega t}$, where the division by $p \cdot \Omega t$ is uniquely defined (as $\Omega^{*}$ of such spaces is a free $\mathbb{L}$-module). By Theorem $3.6, G=\widetilde{G}$. It remains to apply the general (non-additive) Riemann-Roch Theorem - see [15, Proposition 5.19]. Recall, that due to the Projective Bundle axiom, any element of $\Omega^{*}\left(X \times\left(\mathbb{P}^{\infty}\right)^{d}\right)$ can be written as a (unique) $\Omega^{*}(X)$-power series $\alpha\left(z_{1}^{A}, \ldots, z_{d}^{A}\right)$ in the 1 -st Chern classes of the bundles $\mathcal{O}(1)$ from components. The superscripts $A$ is introduced to indicate that we are dealing with the source of the operation. When we apply any operation $F$ to $\alpha\left(z_{1}^{A}, \ldots, z_{d}^{A}\right)$, we obtain again some $\Omega^{*}(X)$-power series in $z_{1}^{B}, \ldots, z_{d}^{B}$ (the same 1-st Chern classes, but in the target) which we denote $F\left(\alpha\left(z_{1}^{A}, \ldots, z_{d}^{A}\right)\right)\left(z_{1}^{B}, \ldots, z_{d}^{B}\right)$. Now we can plug whatever we want instead of the formal $B$-variables. This way, we can describe what happens to $F$ under regular push-forwards. Namely, by [15, Proposition 5.19], the condition $\left(b_{i i}\right)$ of [15, Definition $5.5]$ is satisfied, and so we have, for any $u \in \Omega^{*}(X)$,

$$
\begin{gathered}
F\left(f_{*}(u)\right)=f_{*} \operatorname{Res}_{s=0} \frac{F\left(\prod_{l=1}^{d} z_{l}^{A} \cdot u\right)\left(z_{l}^{B}=s+\left.\Omega \lambda_{l}\right|_{l \in \bar{d}}\right) \cdot \omega_{s}}{\prod_{l=1}^{d}\left(s+\Omega \lambda_{l}\right) \cdot s}, \quad \text { which implies : } \\
\Phi(\bar{i})^{q(t)}\left(f_{*}(u)\right)=f_{*} \operatorname{Res}_{s=0} \frac{\Phi(\bar{i})^{\widetilde{q}(t)}(u)\left(z_{l}=s+\left.\Omega \lambda_{l}\right|_{l \in \bar{d}}\right) \cdot \omega_{s}}{\prod_{l=1}^{d}\left(s+\Omega \lambda_{l}\right) \cdot s}= \\
f_{*} \Phi(\bar{i})^{\widetilde{\widetilde{q}}(t)}(u)\left(z_{l}=\left.\lambda_{l}\right|_{l \in \bar{d}}\right)=f_{*} \Phi(\bar{i})^{q(t) \cdot c^{\Omega}\left(\mathcal{N}_{f} ; \bar{i}\right)}(u) .
\end{gathered}
$$

(Notice, that $\Phi(\bar{i})^{\widetilde{q}(t)}$ is $\Omega^{*}\left(X \times\left(\mathbb{P}^{\infty}\right)^{\times d}\right)$-linear in $\widetilde{q}$, by definition, and so, $\Phi(\bar{i})^{\widetilde{q}(t)}(u)\left(\left.z_{l}\right|_{l \in \bar{d}}\right)=$ $\left.\left(\prod_{l \in \bar{d}} z_{l}\right) \cdot \Phi(\bar{i})^{\widetilde{q}(t)}(u)\left(\left.z_{l}\right|_{l \in \bar{d}}\right).\right)$

As an application of the above result we obtain that Symmetric operations provide obstructions for a cobordism class to be presented by a class of an embedding (cf. [12, Proposition 3.2]).

Proposition 7.4 Let $V \stackrel{v}{\rightarrow} X$ be a regular embedding. Then $\Phi(\bar{i})(v)=0$. 
Proof: By dimensional considerations, $\Phi(\bar{i})\left(1_{\operatorname{Spec}(k)}\right)=0$. Since $\Phi(\bar{i})$ is an operation, we have: $\Phi(\bar{i})\left(1_{V}\right)=0$, for all $V$. Then it follows from Proposition 7.3 that $\Phi(\bar{i})(v)=0$.

In another direction, our Symmetric operations help to study $\mathbb{L}$-torsion and relations in $\Omega^{*}(X)$. For this purpose, let us introduce a close relative of the invariant of M.Rost.

Definition 7.5 Having $p$ and $\bar{i}$, and a smooth projective $U$ of positive dimension, define

$$
\eta_{p, \bar{i}}(U):=-\frac{\operatorname{deg}\left(c\left(-T_{U} ; \bar{i}\right)_{0}\right)}{p} \in \mathbb{Z}\left[\mathbf{i}^{-1}\right],
$$

where $T_{U}$ is the tangent bundle, and $c\left(-T_{U} ; \bar{i}\right)_{0}$ is the zero-dimensional $\left(t^{\left.-p \operatorname{dim}(U)_{-}\right)}\right.$component of the $\prod_{j=1}^{p-1} c\left(-T_{U}\right)\left(i_{j} \cdot t\right)$ (here $c(\mathcal{N})(t)$ is the total Chern power series - see above).

The fact that $\eta_{p, i}$ is well-defined follows from the following result:

Proposition 7.6 Let $U$ be smooth projective variety of dimension $n>0$, and $[U] \in \mathbb{L}$ be the respective class. Then

$$
\operatorname{deg}\left(\Phi(\bar{i})^{t^{p n}}([U])\right)=\eta_{p, \bar{i}}(U) \in \mathbb{Z}\left[\mathbf{i}^{-1}\right] .
$$

Proof: We know that $\Phi(\bar{i})^{t^{p n}}([U]) \in \mathbb{L}_{0}\left[\mathbf{i}^{-1}\right]=\mathbb{Z}\left[\mathbf{i}^{-1}\right]$, and

$$
\begin{aligned}
& p \cdot \Phi(\bar{i})^{t^{p n}}([U])=-\operatorname{Res}_{t=0} \frac{t^{p n} \cdot S t(\bar{i})([U]) \omega_{t}}{t}=-\operatorname{Res}_{t=0} \frac{t^{p n} \cdot S t(\bar{i})\left(\pi_{*}\left(1_{U}\right)\right) \omega_{t}}{t}= \\
& -\operatorname{Res}_{t=0} \frac{t^{n} \cdot-\pi_{*}\left(c\left(-T_{U} ; \bar{i}\right)\right) \omega_{t}}{t}=-\operatorname{deg}\left(c\left(-T_{U} ; \bar{i}\right)_{0}\right),
\end{aligned}
$$

where $\pi: U \rightarrow \operatorname{Spec}(k)$ is the projection.

Remark 7.7 The invariant of M.Rost is defined a bit differently. It is the degree of the zero cycle $c_{1}(\mathcal{L})^{n p}$, where $\mathcal{L}$ is the standard linear bundle on a smooth, but not proper variety $C^{p} U \backslash \Delta(U)$ (here $\mathcal{L}$ is a quotient of $\mathcal{O}$ on $U^{\times p} \backslash \Delta(U)$ by the $\mathbb{Z} / p$-action). Such a degree is well-defined in $\mathbb{Z} / n_{U}$, where $n_{U}$ is the greatest common divisor of the degrees of closed points on $U$.

Composing the Total Landweber-Novikov operation with the canonical morphism of theories $p r: \Omega^{*} \rightarrow \mathrm{CH}^{*}$ and evaluating on a point, we obtain the Hurewitz map

$$
\mathbb{L} \rightarrow \mathbb{Z}\left[b_{1}, b_{2}, \ldots\right] .
$$

Coefficients at particular monomials give us characteristic numbers $\chi_{\bar{b}^{J}}: \mathbb{L} \rightarrow \mathbb{Z}$. On the class of a smooth projective variety $U$ these can be alternatively computed as degrees of zerocycles given by certain polynomials in Chern classes of $-T_{U}$. More precisely, the respective zero-cycle will be the coefficient at $\bar{b}^{\bar{J}}$ in the product $\prod_{\lambda \in \Lambda}\left(1+b_{1} \lambda+b_{2} \lambda^{2}+\ldots\right)$, where $\Lambda$ is the set of Chow-roots of $-T_{U}$. Let $I(p) \subset \mathbb{L}$ be the ideal consisting of classes whose all characteristic numbers are divisible by $p$. Due to results of Landweber, it is stable under 
the action of the Landweber-Novikov operations (which is obvious as soon as we know that Landweber-Novikov operations form an algebra, and that characteristic numbers are exactly the results of various Landweber-Novikov operations applied to the class). I recall, that an element $x \in \mathbb{L}_{p^{r}-1}$ of dimension $\left(p^{r}-1\right)$ is called a $\nu_{r}$-element, if it belongs to $I(p)$, and the (only) additive characteristic number of it is not divisible by $p^{2}$. After projecting to $B P$-theory such an element can be chosen as a polynomial generator of the coefficient ring see [18].

Since $\mathbb{Z}\left[\mathbf{i}^{-1}\right] / p=\mathbb{Z} / p$, we can compare $\bar{\eta}_{p, \bar{i}}(U) \in \mathbb{Z} / p$ for different $\bar{i}$.

Proposition 7.8 (cf. [9])

Let $[U] \in I(p)$. Then, for all $\bar{i}$ and $\bar{l}$,

$$
\bar{\eta}_{p, \bar{i}}(U)=\bar{\eta}_{p, \bar{l}}(U)
$$

(2) Let $U$ has no zero cycles of degree prime to $p$. Then, up to sign, $\bar{\eta}_{p, \bar{i}}(U)$ coincides with $\bar{\eta}_{p}(U)$ - the invariant of M.Rost $\bmod p ;$

(3) If $[U]$ is a $\nu_{r}$-element in $\mathbb{L}$, then $\bar{\eta}_{p}(U) \neq 0$.

Proof: (1) We know that $\left(\gamma_{S t(\bar{i})}-\gamma_{S t(\bar{l})}\right)$ is divisible by $[p]_{\Omega}$. But any multiplicative operation $G: \Omega^{*} \rightarrow B^{*}$ with $\gamma_{G}(x)=b_{0} x+b_{1} x^{2}+\ldots$, where $b_{0}$ is invertible, is a generalized specialization of the Total Landweber-Novikov operation. In particular, $G$ is a linear combination of specializations of the individual Landweber-Novikov operations with coefficients - monomials in $b_{0}^{ \pm 1}$ and $b_{k} / b_{0}, k>0$. Thus $(S t(\bar{i})-S t(\bar{l}))$ will be a linear combination of the LandweberNovikov operations with coefficients divisible by $[p]_{\Omega}$ (note, that this linear combination will depend on the component $\Omega^{n}$ on which it acts, as our operation is unstable). This implies that the difference of two Symmetric operations

$$
(\Phi(\bar{i})-\Phi(\bar{l})): \Omega^{*} \rightarrow \Omega^{*}\left[\mathbf{i}^{-1}, \mathbf{l}^{-1}\right]\left[t^{-1}\right]
$$

is a linear combination of the Landweber-Novikov operations. It remains to observe that the ideal $I(p)$ is stable under the latter, and the zero-dimensional component of $I(p)$ is $p \cdot \mathbb{Z}$.

(2) If $U$ has no zero cycles of degree prime to $p$, then we have the surjection $\mathbb{Z} / n_{U} \rightarrow \mathbb{Z} / p$ (notice, that, in particular, $[U] \in I(p)$ ). It follows from computations of M.Rost in [9] that $-\mathbf{i}^{\operatorname{dim}(U)} \cdot \bar{\eta}_{p, \bar{i}}(U)=\bar{\eta}_{p}(U)$. Remains to observe that $\mathbf{i} \equiv-1(\bmod p)$.

(3) Since $\prod_{j=1}^{p-1}(x+j t) \equiv \prod_{j=1}^{p-1}\left(x+\varepsilon^{j} t\right) \equiv\left(x^{p-1}+t^{p-1}\right)(\bmod p)$, where $\varepsilon$ is a primitive root of 1 of degree $(p-1)$, by the considerations from part (1), we obtain that the characteristic number for $c\left(-T_{U} ; \bar{i}\right)$ can be substituted by the one for $b\left(-T_{U}\right)$, where $b(\mathcal{N})(t)=$ $\prod_{j=1}^{p-1} c(\mathcal{N})\left(\varepsilon^{j} \cdot t\right)$. Notice, that $\left(b\left(-T_{U}\right)\right)_{0}$ is the characteristic number corresponding to the partition $(p-1, p-1, \ldots, p-1)$, or in other words, to the Landweber-Novikov operation $S_{L-N}^{b_{p-1}^{d}}$, where $d=\frac{\operatorname{dim}(U)}{p-1}$. The latter operation is a component of the multiplicative operation

$$
\sum_{m \geqslant 0} b_{p-1}^{m} \cdot S_{L-N}^{b_{p-1}^{m}}: \Omega^{*} \rightarrow \Omega^{*}\left[b_{p-1}\right]
$$


(a specialization of the Total Landweber-Novikov operation: $b_{i} \mapsto 0, i \neq p-1$ ). And operations $S_{L-N}^{b_{p-1}^{m}}$ descend to Steenrod operations on $\mathrm{CH}^{*} / p$, which implies that $\chi_{b_{p-1}^{m}}$ is always divisible by $p$, for $m>0$. It follows that our characteristic number $\chi_{b_{p-1}^{d}}$ is divisible by $p^{2}$ on every decomposable element of the Lazard ring. Hence, modulo $p^{2}$ it is the same (up to a factor invertible modulo $p$ ) on each $\nu_{r}$-element (since such an element can be chosen as a polynomial generator of $\left.\mathbb{L} \otimes \mathbb{Z}_{(p)}\right)$. It is sufficient to compute it on the class of a hypersurface $Q$ of degree $p$ in $\mathbb{P}^{p^{r}}$. And for such a hypersurface, $\bar{\chi}_{b_{p-1}^{d}}(Q)=(-1)^{r}\left(\begin{array}{c}\frac{p^{r+1}-1}{p-1} \\ \frac{p^{r}-1}{p-1}\end{array}\right) \neq 0 \in \mathbb{Z} / p \mathbb{Z}$.

The above invariants can be applied to the computation of Chow traces $\phi(\bar{i})^{q(t)}$ of Symmetric operations, that is, compositions $\Omega^{*} \stackrel{\Phi(\bar{i})^{q(t)}}{\longrightarrow} \Omega^{*} \stackrel{p r}{\longrightarrow} \mathrm{CH}^{*}$. In analogy with slices of $\Phi$, for any $f(t) \in \mathbb{L}[[t]]\left[t^{-1}\right]$, let us denote as $S t(\bar{i})^{f(t)}$ the operation $\operatorname{Res}_{t=0} \frac{f(t) \cdot S t(\bar{i}) \cdot \omega_{t}}{t}$, and as $s t(\bar{i})^{f(t)}$ the Chow trace of it.

The following result shows that if we are given $u \cdot v$, where $u \in \mathbb{L}_{>0}$, then using Symmetric operations we can obtain, if not $v$ itself, at least, some multiple of it's Chow trace $\operatorname{pr}(v)$. And the coefficient involved is invertible modulo $p$ in interesting situations (which will not be the case if one uses Landweber-Novikov, or Steenrod operations instead).

Proposition 7.9 Let $v \in \Omega^{*}(X)$ and $u=[U] \in \mathbb{L}_{>0}$. Let $q(t) \in \mathrm{CH}^{*}(X)[[t]]$. Then

$$
\phi(\bar{i})^{q(t)}(u \cdot v)=\eta_{p, \bar{i}}(U) \cdot s t(\bar{i})^{q(t) t^{-p \cdot \operatorname{dim}(U)}}(v) .
$$

In particular, if $k=p \operatorname{dim}(u)-(p-1) \operatorname{codim}(v)$ is positive, then

$$
\phi(\bar{i})^{t^{k}}(u \cdot v)=\eta_{p, \bar{i}}(U) \cdot \mathbf{i}^{\operatorname{codim}(v)} \cdot \operatorname{pr}(v)
$$

Proof: Since both sides of the equation are $\mathrm{CH}^{*}(X)$-linear on $q(t)$, we can assume that $q(t) \in \mathbb{Z}[[t]]$. Then it follows from Theorem 3.6 that it is sufficient to compare our operations (as operations on $v$ ) on cellular spaces $\left(\mathbb{P}^{\infty}\right)^{\times l}$. On such a space, using the fact that $S t(\bar{i})$ is multiplicative, we can write LHS as

$$
\begin{aligned}
& -p r \operatorname{Res}_{t=0} \frac{S t(\bar{i})(u \cdot v) \cdot q(t) \cdot \omega_{t}}{p \cdot \Omega t}=-\operatorname{Res}_{t=0} \frac{\operatorname{pr}\left(S t(\bar{i})(u) \cdot S t(\bar{i})(v) \cdot q(t) \cdot \omega_{t}\right)}{p \cdot t}= \\
& \eta_{p, \bar{i}}(U) \cdot \operatorname{Res}_{t=0} \frac{s t(\bar{i})(v) \cdot q(t) \cdot d t}{t^{p \cdot \operatorname{dim}(u)+1}}=\operatorname{RHS}
\end{aligned}
$$

The second equality follows from the fact that $\gamma_{s t(\bar{i})}(x)=x \cdot\left(\mathbf{i} \cdot t^{p-1}\right)+\ldots+x^{p}$, and so

$$
s t(\bar{i})^{t^{-(p-1) \operatorname{codim}(v)}}(v)=\mathbf{i}^{\operatorname{codim}(v)} \cdot \operatorname{pr}(v) .
$$


Remark 7.10 Similar result can be obtained with the help of Landweber-Novikov (or Steenrod) operations, but then the number $\eta_{p, \bar{i}}(U)$ will be substituted by $p \cdot \eta_{p, \bar{i}}(U)$, and such a difference is crucial for p-torsion elements. This subtlety of Symmetric operations comes from the fact that these operations encode p-divisibility of certain characteristic numbers, and in reality, all p-primary divisibilities of characteristic numbers are controlled by compositions of Symmetric operations related to $p$.

As an illustration we have:

Corollary 7.11 Let $u \in \mathbb{L}_{p^{r}-1}$ be a $\nu_{r}$-element, and $v \in \Omega^{m}(X)$, where $m<\frac{p\left(p^{r}-1\right)}{p-1}$. Then

$$
u \cdot v=0 \Rightarrow \operatorname{pr}(v)=0 \in \mathrm{CH}^{*}(X) \otimes \mathbb{Z}_{(p)} .
$$

Proof: It follows from Proposition 7.9 that $\eta_{p, \bar{i}}(u) \cdot \operatorname{pr}(v)=0 \in \mathrm{CH}^{*}(X)\left[\mathbf{i}^{-1}\right]$, where $\eta_{p, \bar{i}}(u) \in$ $\mathbb{Z}\left[\mathbf{i}^{-1}\right]$ is relatively prime to $p$ by Proposition 7.8 .

The above result can be used, for example, to compute the Algebraic Cobordism theory and Chow groups of a Rost motive and a Pfister quadric - see [12, the proof of Theorem 4.1]. The same methods give the computation of the Algebraic Cobordism of a generalized Rost motive (an analogue of the Rost motive for $p>2$ ).

The operation $\Phi=\Phi(\bar{i})$ is not additive, but is very close to such:

\section{Proposition 7.12}

$$
\Phi(u+v)=\Phi(u)+\Phi(v)+f_{p}(u, v),
$$

where $f_{p}(u, v)=\sum_{l=1}^{p-1} \frac{\left(\begin{array}{l}p \\ l\end{array}\right)}{p} u^{l} v^{p-l}$ is a polynomial of degree $p$ in $u, v$.

Proof: For cellular spaces, where the division by $[p]_{\Omega}=\frac{p \cdot \Omega}{t}$ is well-defined, the statement follows directly from the definition of $\Phi$ and the fact that $S t$ is additive. The general case follows from Theorem 3.6 considering the external $\left(u \in \Omega^{*}(X), v \in \Omega^{*}(Y)\right)$ version of the statement.

The following statement describes the multiplicative properties of the Total Symmetric operation.

Proposition 7.13 Let $u, v \in \Omega^{*}(X)$. Let $S t=S t(\bar{i}), \Phi=\Phi(\bar{i})$, and $[p]_{\Omega}=\frac{p \cdot \Omega t}{t}$. Then

$$
\Phi(u \cdot v)=\left(\Phi(u) \cdot S t(v)+S t(u) \cdot \Phi(v)+\Phi(u) \Phi(v) \cdot[p]_{\Omega}\right)_{\leqslant 0}
$$

Proof: Considering the external version of this statement $\left(u \in \Omega^{*}(X), v \in \Omega^{*}(Y)\right)$, and fixing $u$ (respectively $v$ ), we obtain from Theorem 3.6 that it is sufficient to check our statement for $\left(\mathbb{P}^{\infty}\right)^{\times l}$, for all $l$. We know that $S t(u)=\delta(u)+\square^{p}(u)-\Phi(u) \cdot[p]_{\Omega}$, for some $\delta(u) \in \Omega^{*}(X)[[t]] t$, and similar for $v$. Using the multiplicative property of $S t$ we get:

$$
\square^{p}(u \cdot v)-S t(u \cdot v)=\left(\Phi(u) \cdot S t(v)+S t(u) \cdot \Phi(v)+\Phi(u) \cdot \Phi(v) \cdot[p]_{\Omega}\right) \cdot[p]_{\Omega}+\delta^{\prime},
$$


where $\delta^{\prime} \in \Omega^{*}(X)[[t]] t$. Since now $X$ is cellular, the division by $[p]_{\Omega}$ is unique, and we get:

$$
\Phi(u \cdot v)=\left(\Phi(u) \cdot S t(v)+S t(u) \cdot \Phi(v)+\Phi(u) \Phi(v) \cdot[p]_{\Omega}\right)_{\leqslant 0} .
$$

The action of the Symmetric operations simplifies substantially in the case of the Graded Algebraic Cobordism. Recall, that $G r \Omega^{*}=\oplus_{r} \geqslant 0 \Omega_{(r)}^{*}$, where $\Omega_{(r)}^{*}(X)=F^{r} \Omega^{*}(X) / F^{r+1} \Omega^{*}(X)$, and $F^{r} \Omega^{*}(X)$ consists on elements supported on closed subschemes of codimension $\geqslant r$ (that is, vanishing on open complements to such subschemes). Any cohomological operation preserves the support of the element, and so acts on the graded theory as well. We have natural surjection of $\mathbb{L}$-modules: $\mathrm{CH}^{r}(X) \otimes_{\mathbb{Z}} \mathbb{L} \rightarrow \Omega_{(r)}^{*}(X)$, which we simply denote as $z \otimes u \mapsto z \cdot u$. The action of Steenrod and Symmetric operations in the graded case can be described as follows (as above, we drop $(\bar{i})$ from the notations):

Proposition 7.14 Let $z \in \mathrm{CH}^{r}(X), u \in \mathbb{L}_{d}$. Then:

1) $S t(z \cdot u)=z \cdot t^{r(p-1)} \cdot \mathbf{i}^{r} \cdot S t(u)$;

2) $\Phi(z \cdot u)=z \cdot t^{r(p-1)} \cdot \mathbf{i}^{r} \cdot \Phi(u)_{\leqslant-r(p-1)}$, where $r \neq 0$.

Proof: The case $r=0$ is trivial. For $r>0$, since we are working modulo elements supported in codimension $\geqslant(r+1)$, we can assume that $z$ is represented by a regular embedding, which gives that $S t(z)=z \cdot \mathbf{i}^{r} \cdot t^{r(p-1)}$. Then part 1) follows from the multiplicativity of $S t$.

The second part follows from Proposition 7.13 taking into account that $\Phi(z)=0$ (see Proposition (7.4).

Using the action of Symmetric operations on $G r \Omega^{*}$ we prove in [16, Therem 4.3] that the Algebraic Cobordism $\Omega^{*}(X)$ as a module over $\mathbb{L}$ has relations in positive codimensions (and the same holds for the graded version). This extends the result of M.Levine-F.Morel 4, Theorem 4.4.7] claiming that the generators of this $\mathbb{L}$-module are in non-negative codimensions.

This, in particular, gives the computation of the Algebraic Cobordism of a curve:

$$
\Omega^{*}(C)=\left(\mathbb{L} \otimes_{\mathbb{Z}} \operatorname{Pic}(C)\right) \oplus \mathbb{L} \cdot 1_{C}
$$

\section{References}

[1] P.Brosnan, Steenrod operations in Chow theory, Trans. Amer. Math. Soc. 355 (2003), no.5, 1869-1903.

[2] T. tom Dieck, Steenrod-operationen in Kobordismen-Theorien., Math. Z. 107 (1968), 380-401.

[3] T.Kashiwabara, Hopf rings and unstable operations, J. of Pure and Applied Algebra 94, 1994, 183-193 
[4] M.Levine, F.Morel, Algebraic cobordism, Springer Monographs in Mathematics, Springer-Verlag, 2007.

[5] M.Levine, R.Pandharipande, Algebraic cobordism revisited, Invent. Math., 176 (2009), no. $1,63-130$.

[6] I.Panin, Oriented Cohomology Theories of Algebraic Varieties, K-theory J., 30 (2003), 265-314.

[7] I.Panin, A.Smirnov, Push-forwards in oriented cohomology theories of algebraic varieties, K-theory preprint archive, 459, 2000. http://www.math.uiuc.edu/K-theory/0459/

[8] D.Quillen, Elementary proofs of some results in cobordism theory using Steenrod operations, Adv. Math, 7 (1971), 29-56.

[9] M.Rost, Notes on the degree formula, Preprint, December 2001; available at: http://www.math.uni-bielefeld.de/ rost/degree-formula.html

[10] A.Smirnov, Orientations and transfers in cohomology of algebraic varieties, St. Petersburg Math. J., 18, n.2, (2007), 305-346.

[11] A.Vishik, Symmetric operations (in Russian), Trudy Mat. Inst. Steklova 246 (2004), Algebr. Geom. Metody, Svyazi i Prilozh., 92-105. English transl.: Proc. of the Steklov Institute of Math. 246 (2004), 79-92.

[12] A.Vishik, Symmetric operations in Algebraic Cobordism, Adv. Math, 213 (2007), 489552 .

[13] A.Vishik, Generic points of quadrics and Chow groups, Manuscripta Math 122 (2007), No.3, 365-374.

[14] A.Vishik, Stable and Unstable Operations in Algebraic Cobordism, arXiv:1209.5793 [math.AG], 25 Sep. 2012, 65 pages.

[15] A.Vishik, Operations and poly-operations in Algebraic Cobordism, arXiv:1409.0741 [math.AG], 2 Sep. 2014, 26 pages.

[16] A.Vishik, Algebraic Cobordism as a module over the Lazard ring, arXiv:1409.0156 [math.AG], 30 Aug. 2014, 8 pages.

[17] V.Voevodsky, Reduced power operations in motivic cohomology, Publ. Math. IHES 98 (2003), 1-57.

[18] W.S.Wilson, Brown-Peterson homology: an introduction and sampler, Conference Board of the Mathematical Sciences, Regional Conference Series in Mathematics, 48, AMS, Providence, Rhode Island, 1980, 1-86. 